\title{
Vulnerability Analysis of Fire Spreading in a Building using Fuzzy Logic and its Integration in a Decision Support System
}

\author{
Sanae KHALI ISSA \\ Laboratory of Computer Sciences, \\ Systems and Telecommunication \\ Faculty of Sciences and Technology \\ Tangier, Morocco
}

\author{
Abdellah AZMANI \\ Laboratory of Computer Sciences, \\ Systems and Telecommunication \\ Faculty of Sciences and Technology \\ Tangier, Morocco
}

\author{
Benaissa AMAMI \\ Laboratory of Computer Sciences, \\ Systems and Telecommunication \\ Faculty of Sciences and Technology \\ Tangier, Morocco
}

\begin{abstract}
In this paper, a new method is proposed to analyze the vulnerability of fire spreading in building. This study is based on the application of the artificial intelligence using fuzzy logic.

After an overview of the phenomenon of spreading [1] [2], an identification of the various parameters that may influence this phenomenon has been achieved and by applying fuzzy logic technique, the vulnerability analysis has been established.
\end{abstract}

This study represents an important step for modeling a decision support system in order to optimize the fire risk in a building.

\section{General Terms}

Making decision, artificial intelligence, fire security.

\section{Keywords}

Artificial intelligence, fuzzy set, vulnerability, spreading, fire risk.

\section{INTRODUCTION}

The fire is considered as one of the critical problems that can disrupt human life. It can be violent and destructive for human activities and nature. In fact, it is an uncontrolled reaction in space and time [3].

In the article [4] published previously, a method has been proposed for a predictive management of fire spreading risk in a building, this predictive management will be integrated into a decision support system. This study was based on decision trees. Furthermore, fire spreading impact on human life has been identified by applying Bayesian Networks [5][6][7]. This method is based on an exhaustive identification of different parameters that increase or reduce the risk:

- The presence or absence of flammable materials;

- The presence or absence of combustible components;

- The state of the doors and the windows (open or closed);

- The nature of the building walls (fire resistance).
The obtained results show clearly the probability increase of fire spreading as a function of some parameters presence favoring this phenomenon (Figure 1).

Fire spreading probability

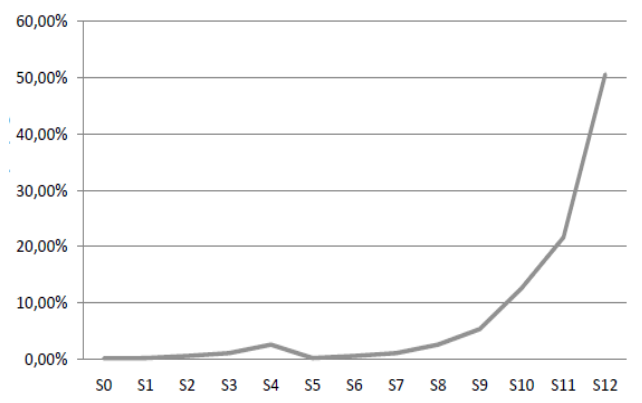

Fire spreading degree (Presence of parameters)

Fig.1: Probability variation of fire spreading as a function of the favoring parameters presence

For the second obtained result, the number of humans injured increases gradually as a function of the degree of fire spreading (figure 2).

Number of human injury

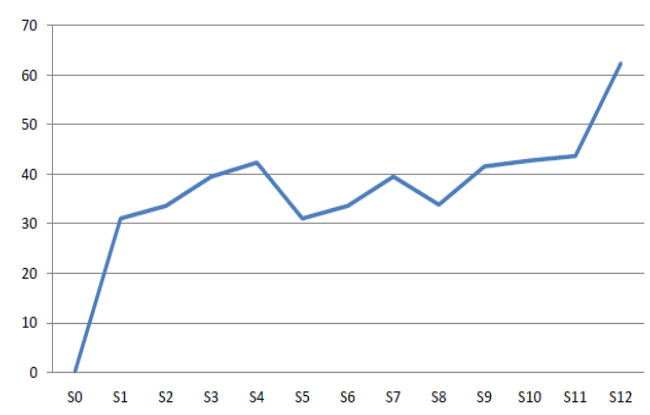

Fire spreading degree (Presence of parameters)

Fig.2: Variation of injured people number as a function of the fire spreading degree 


\section{FIRE AND ITS PROBLEMATIQUE}

\subsection{General description}

Fire is an exothermic phenomenon which needs three parameters:

- $\quad$ Fuel (solid, liquid or gaseous);

- Oxygen;

- Heat source

This reaction can be represented as a schema called fire triangle:

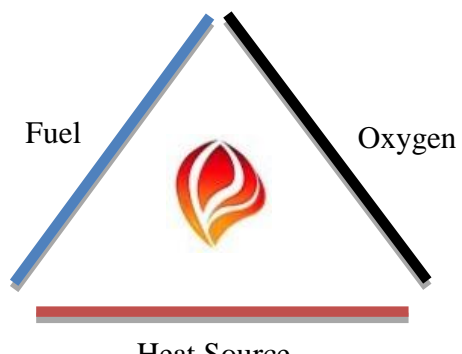

Heat Source

Fig.3: Fire triangle [8]

\subsection{Steps of fire development}

After many studies done about the fire security in building [9][10], we found that the development process of the fire can be divided into four phases [11]:

- Startup of fire: during this phase, the fuel begins to burn, the temperature is located at the point of ignition and the first gas and smoke appear.

- Outbreak: the flames radiation achieves the proximate materials and the hot gases are released and fill out all the volume.

- Flashover: during this step, the fuel achieves its ignition temperature, the volume is embarrassed and fire reaches its maximum

- Fallen fire: the fuel disappears gradually and the temperature drops.

\subsection{Parameters affecting the fire spreading}

The vulnerability of fire spreading in a building may depend on two types of factors [12] [13][14][15]:

\subsubsection{Factors related to the construction of the} structure, source of fire:

- Volume of the space (height and area);

- Oxygen flow in the space;

- Area and number of open doors and windows;

- Type of doors and windows (fire doors or normal);

- Thickness of walls;

- Flammability of building materials;

- Flammability degree of building materials.

\subsubsection{Factors related to products and equipments present in the building:}

- Quantity of the products;

- Combustion speed of the products;

- Flammability of the products.

- Flammability degree of the products.

\section{PROPOSED METHOD}

\subsection{General principle}

The fire spreading is a phenomenon which depends on many factors, the most important ones are: the amount of oxygen existing in the construction, source of fire and the combustion speed of existing combustible materials.

Therefore, the disappearance of fuel or oxygen can stop immediately the fire.

The method proposed in this article is to model the fire spreading time in a building as a function of the oxygen quantity and the combustion speed of combustible materials present therein.

This study is based on the application of fuzzy logic technique [16][17] which is one of most used decision making methods.

The choice of this technique is justified by the nature of variables to be modeled. They are linguistic variables which vary in a set of intervals and which take different values according to the building to be studied.

\subsection{Application process}

To apply the proposed method, the steps below must be followed:

- Identification of linguistic variables of the system (input and output variables);

- Specification of the universes of discourse variables;

- Identification of the membership functions for each variable;

- Definition of the system operating rules;

- Computation of the membership functions for the output variables;

- $\quad$ Selection and application of an inference method;

- Application of a method of defuzzification;

- Results analysis.

\section{OVERVIEW ON FUZZY LOGIC}

Fuzzy logic is a technique of artificial intelligence based on fuzzy set theory [18][19].

It is a tool for decision making in situations of uncertainty where there is a lack of accurate and reliable mathematical probabilities. Thereby, it is an adaptive technique for evolutive situations.

Fuzzy logic is used to guide the control systems and to model the variation of some variables as a function of others.

The operation of this technique is described by the following diagram: 


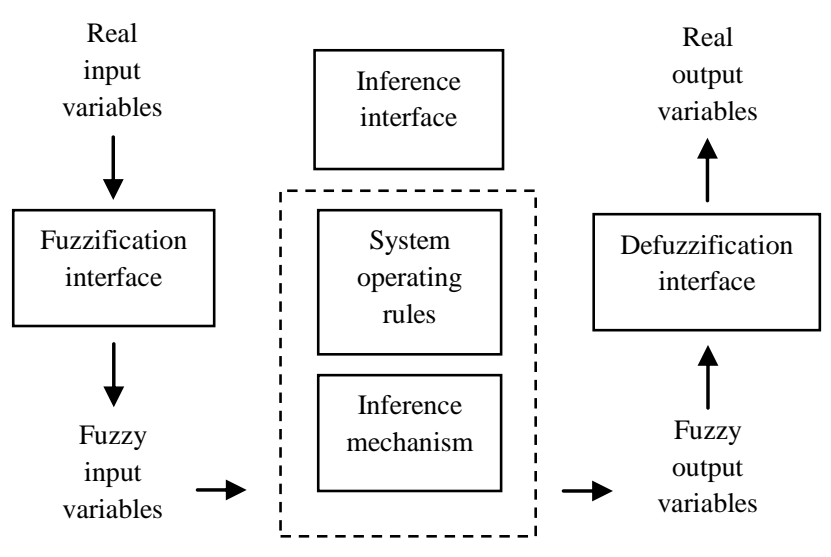

Fig.4: Process of fuzzy logic technique [18]

There are many libraries which implement the fuzzy set technique as $\mathrm{C}++$, java, python, Matla, in this paper, we choose the fuzzy logic toolbox in Matlab [20].

\section{CASE STUDY}

\subsection{Identification of linguistic variables and their values}

\subsubsection{Input variables:}

According to questionnaires proposed to some experts in fire safety domain as well as to those of the fuzzy logic domain, the determination of the various fuzzy variables is established in the following way:

OxygenVolume to indicate the oxygen quantity present in the construction, source of fire.

The volume of oxygen (OxygenVolume) possesses as linguistic values: Low, Medium and High distributed on a universe of discourse varying between 0 and $10 \mathrm{~m} 3$.

CombustionSpeed to designate the combustion speed of combustible materials present in the construction at time of fire ignition.

The combustion speed (CombustionSpeed) has as linguistic values: Slow, Medium and Fast which are distributed on a universe of discourse going from 0 to $2 \mathrm{~mm} / \mathrm{min}$.

\subsubsection{Output variables:}

FireDuration to indicate the fire spreading duration in the building.

The fire duration (FireDuration) has as linguistic values: Short, Meduim and Long.

The universe of discourse of the variable FireDuration varies from 0 to $120 \mathrm{~min}$.

\subsection{Identification of the membership functions}

The membership functions used has a trapezoidal form because it is the most appropriate for the variables selected in this study.

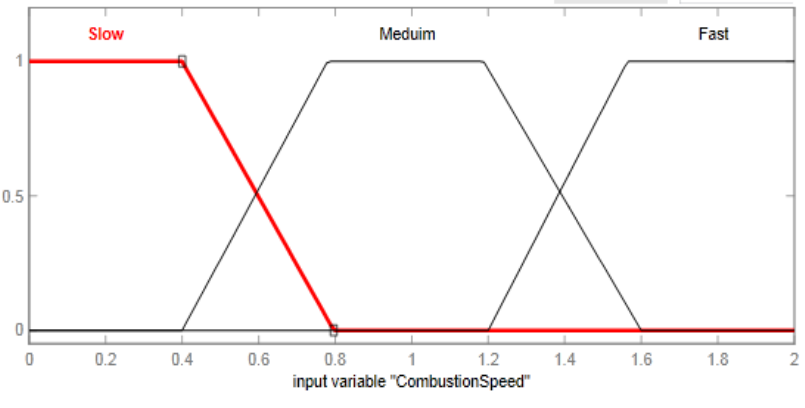

Fig.5: The membership function of the variable CombustionSpeed

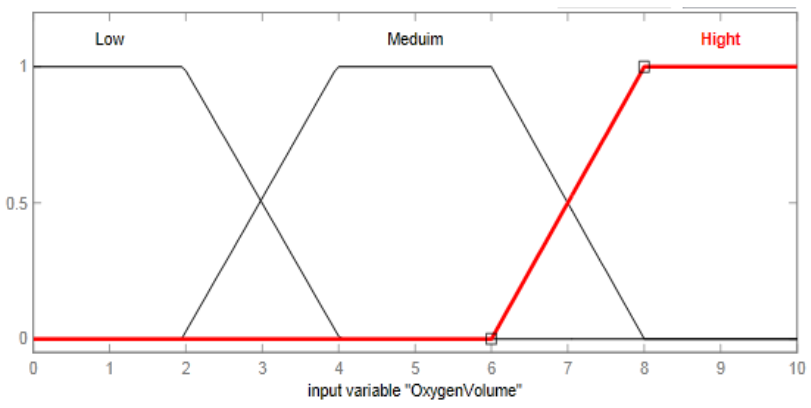

Fig.6: The membership function of the variable OxygenVolume

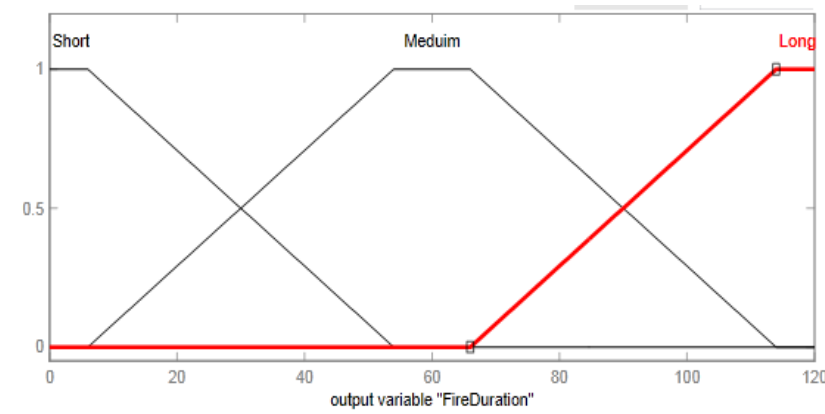

Fig.7: The membership function of the variable FireDuration

\subsection{Definition of the system operating rules}

The basis of rules in this study is attributed by some experts in the fire safety domain and it is presented below:

R1 : If (OxygenVolume is Low) and (CombustionSpeed is Slow) Then (FireDurationis Short)

R2 : If (OxygenVolume is Low) and (CombustionSpeed is Meduim) Then (FireDuration is Short)

R3 : If (OxygenVolume is Low) and (CombustionSpeed is Fast) Then (FireDuration is Meduim)

R4 : If (OxygenVolume is Meduim) and (Combustionspeed is Slow) Then (FireDuration is Short)

R5 : If (OxygenVolume is Meduim) and (Combustionspeed is Meduim) Then (FireDuration is Meduim)

R6 : If (OxygenVolume is Meduim) and (Combustionspeed is Fast) Then (FireDuration is Meduim) 
R7 : If (OxygenVolume is High) and (CombustionSpeed is Slow) Then (FireDuartion is Short)

R8 : If (OxygenVolume is High) and (CombustionSpeed is Meduim) Then (FireDuration is Meduim)

R9 : If (OxygenVolume is High) and (CombustionSpeed is Fast) Then (FireDuration is Long)

These bases of rules will be enriched.

\subsection{Obtained results}

The obtained result is based on the application of the Mamdani inference rule. Its principle is:

Oxygen Volume $=7$

3

4
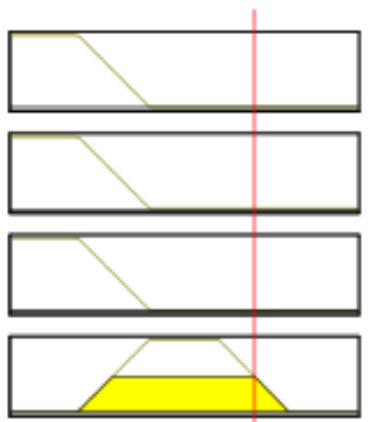

5

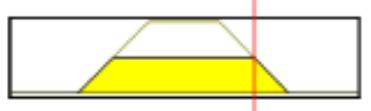

6

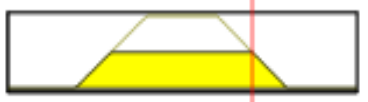

7

8
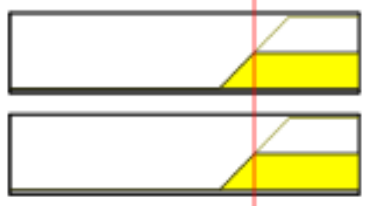

9

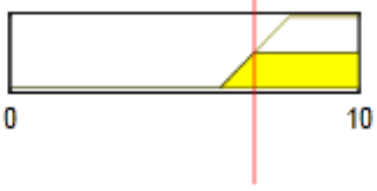

Combustionspeed $=2$
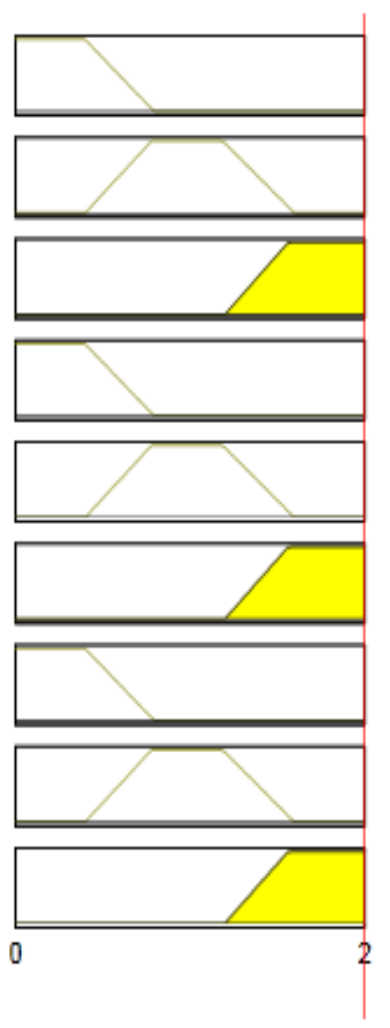

FireDuration $=69.1$
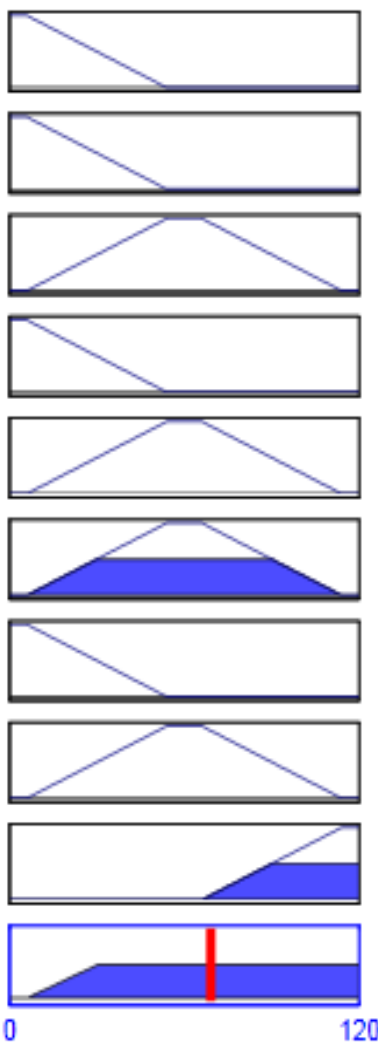

Fig.9: Variation of the fire duration as a function of the oxygen volume and the combustion speed. 


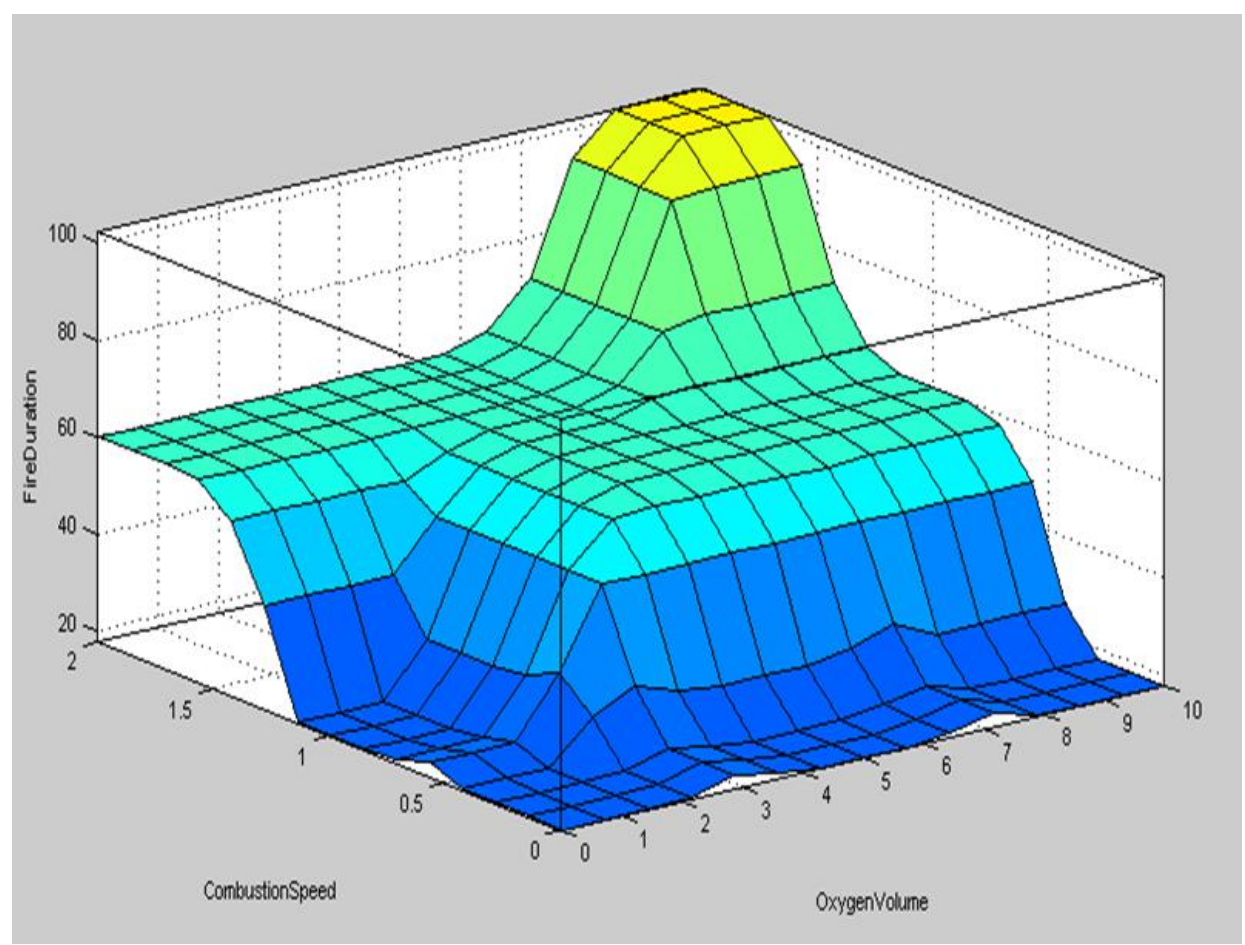

Fig. 10: The surface variation of the fire duration as a function of the oxygen volume and the combustion speed.

\subsection{Results analysis}

The following results allow to specify factors presenting a major interest in the fire spreading phenomenon. This interest will aim for determining the appropriate tool of fire security.

By fixing for example the oxygen volume, and by varying the combustion speed of the present material, the fire duration increases gradually until the total disappearance of fuel as it is mentioned in the following table:

Table 1: Variation of the fire duration as a function of the combustion speed of existing materials in the construction, source of fire.

\begin{tabular}{|c|c|c|}
\hline $\begin{array}{c}\text { Volume of } \\
\text { oxygen }(\mathbf{m 3})\end{array}$ & $\begin{array}{c}\text { Combustion Speed } \\
(\mathbf{m m} / \mathbf{m i n})\end{array}$ & $\begin{array}{c}\text { Fire duration } \\
(\mathbf{m i n})\end{array}$ \\
\hline 5 & 0,3 & 17,8 \\
\hline 5 & 0,7 & 57,2 \\
\hline 5 & 1,8 & 60 \\
\hline
\end{tabular}

Consequently, the proposed tool of security will have for aim to slow down the combustion speed of the present combustible products.

And by fixing the combustion speed, and by varying the oxygen volume, the fire duration increases as it is shown in the following table:
Table 2: Variation of the fire duration as a function of the volume of oxygen present in the construction, source of fire

\begin{tabular}{|c|c|c|}
\hline $\begin{array}{c}\text { Volume of } \\
\text { oxygen (m3) }\end{array}$ & $\begin{array}{c}\text { Combustion Speed } \\
(\mathbf{m m} / \mathbf{m i n})\end{array}$ & $\begin{array}{c}\text { Fire duration } \\
(\mathbf{m i n})\end{array}$ \\
\hline 1 & 0,9 & 18,9 \\
\hline 4 & 0,9 & 59,9 \\
\hline 9 & 0,9 & 60 \\
\hline
\end{tabular}

As a result, the proposed security equipment must reduce the quantity of the oxygen existing in the building.

The solution proposed then is to use security equipment aiming to minimize the available oxygen, thus the combustion reaction of existing products goes slow, in other words, their speed combustion slows down.

\section{CONCLUSION \& PERSPECTIVES}

The study presented in this paper represents another interesting step in the modeling of a decision making system in the fire safety domain. This system will be used by experts to audit existing buildings with the aim of reducing the fire risk. 
The proposed decision support tool will have for inputs the descriptive information about the building to audit (volume of rooms, number of the open doors and windows, type and nature of existing equipment, etc...) and as outputs the vulnerability degree of the fire spreading (described by the fire duration as well as the rate of human and material damage).

In the same way, other studies will be realized by taking into account other influencing factors of the phenomenon of the fire spreading in order to complete the reasoning basis of our decision making tool.

Also, a modeling of the fire risk impact in building by the application of the same technique (fuzzy set) will be established.

\section{REFERENCES}

[1] J. Michel d'Hoop, Sécurité incendie bâtiment, Référence SE2050, Date de publication : 10 avr. 2002.

[2] Grandjean, P. Jouve, La sécurité incendie dans les bâtiments recevant du public, Paris (Fr.) : Editions du Moniteur, 1984. 224 p.

[3] Hajira Bakkali, Sanae Khali Issa, Abdellah Azmani, The modeling of fire hazards in a goods warehouse by the Bayesian network, International Journal of Emerging Trends in Engineering and Development, 2249-6149, March 2013

[4] Sanae Khali Issa, Abdellah Azmani, Karima Zejli : Approche de modélisation de la propagation de l'incendie dans un édifice et son intégration dans un système décisionnel, colloque international Veille Stratégique Scientifique et Technologique (VSST), Ajaccio France, 25 Mai 2012.

[5] P. Naïm, P.H. Wuillemin, Ph. Leray, O. Pourret, A. Becker, Les Réseaux Bayésiens, Groupe Eyrolles, 2004.

[6] B. Bouzy, Réseaux Bayésiens, 26 février 2008.

[7] H. Cheng, G.V. .Hadjisophocleous: The modeling of fire spread in buildings by Bayesian network, Fire Safety Journal 44 901-908, 2009.
[8] J. Chorier, Diagnostic et évaluation des risques incendie d'une construction et de sa mise en sécurité, Université de Savoie, France, 8 Février 2007.

[9] S. R. Bishop and P. G. Holborn, Dynamic modelling of building fires, Appl. Math. Modelling, 1993, Vol. 17, April

[10] Hao Cheng, George V. Hadjisophocleous, Dynamic modeling of fire spread in building, Fire Safety Journal 46(2011)211-224

[11] L. Thomas, G. Archambault, Sécurité incendie, Edition 2005.

[12] Sanae Khali Issa, Abdellah Azmani, Karima Zejli: Predictive Management of Fire Risks in Building, International Journal of Computer Applications 58(15):711, November 2012.

[13] H. Cheng, G.V. Hadjisophocleous: The modeling of fire spread in buildings by Bayesian network, Fire Safety Journal 44 901-908, 2009.

[14] Margrethe Kobes, Ira Helsloot, Bauke de Vries, Jos G. Post, Building safety and human behaviour in fire: A literature review, Fire Safety Journal, Volume 45, Issue 1, January 2010, Pages 1-11, ISSN 0379-7112

[15] Sanae Khali Issa, Abdellah Azmani, Benaissa Amami, Gestion prédictive des risques d'incendies, publié au TELECOM2011 \& 7ème JFMMA, Tanger MAROC, Mars 16-18, 2011

[16] VilémNovák, Jiří Močkoř, Irina Perfilieva, Mathematical principles of fuzzy logic, Boston, Mass. [u.a.]: Kluwer Academic, 1999.

[17] Kwang H. Lee, First course on Fuzzy Theory and Applications, Springer, 7 October 2004

[18] Robert Fuller, Neural Fuzzy Systems, ISBN 951-650624-0, ISSN 0358-5654, Abo 1995.

[19] L.A.Zadeh, Outline of a new approach to the analysis of complex systems and decision processes, IEEE Transactions on Systems, Man and Cybernetics SMC-3, 28-44, 1973

[20] Fuzzy logic toolbox for user with Matlab, MathsWorks R2013a. 